\title{
Meson Production Experiments at CLAS
}

\author{
S. Strauch for the CLAS Collaboration \\ University of South Carolina, Columbia, SC 29208, USA
}

\begin{abstract}
Electromagnetic meson production is an important tool in the investigation of the structure of the nucleon. Consequently, a series of meson photo- and electroproduction experiments have been performed with the CLAS detector at Jefferson Lab. In this overview I will report on measurements of cross sections, as well as recent and upcoming measurements of single- and doublepolarization observables in meson photoproduction. The data will greatly constrain partial-wave analyses and reduce model-dependent uncertainties in the extraction of nucleon resonance properties.
\end{abstract}

Keywords: Photoproduction reactions, Meson production, Polarization

PACS: $25.20 . \mathrm{Lj}$, 13.60.Le, 13.88.+e

This is an overview of meson-production experiments using the CEBAF Large Acceptance Spectrometer (CLAS) [1] at Jefferson Lab. The CLAS detector provides efficient detection of neutral and charged particles over a good fraction of the full solid angle. In the following I restrict myself to meson photoproduction experiments aimed for the study of excited states of the baryon. I will not further discuss other CLAS mesonproduction experiments like the photoproduction of the $\rho^{0}, \omega$, and $\phi(1020)$ mesons on the proton at large momentum transfer [2, 3, 4], the photoproduction of light vector mesons off nulcei in the search for medium modification [5], nor CLAS meson electroproduction data $[6,7]$; both, the latter two topics, were presented during this conference.

The CLAS Collaboration has measured various reaction channels with unpolarized, circularly polarized and linearly polarized real photons on an unpolarized target over a wide energy range. An overview over these experiments with already published results is given in Table 1.

TABLE 1. Published CLAS meson-photoproduction baryonspectroscopy data; not including pentaquark searches $[8,9,10,11$, $12,13]$.

\begin{tabular}{lccc}
\hline Reaction & Cross section & $\begin{array}{r}\text { Single } \\
\text { polarization }\end{array}$ & $\begin{array}{r}\text { Double } \\
\text { polarization }\end{array}$ \\
\hline$\gamma p \rightarrow \pi N$ & $*$ & - & - \\
$\gamma p \rightarrow \eta p$ & {$[14]$} & - & - \\
$\gamma p \rightarrow \eta^{\prime} p$ & {$[15]$} & - & - \\
$\gamma p \rightarrow K Y$ & {$[16,17]$} & {$[18]$} & {$[19]$} \\
$\gamma p \rightarrow \pi^{+} \pi^{-} p$ & - & {$[20]$} & - \\
$\gamma p \rightarrow K^{+} K^{+} \Xi^{-}$ & {$[21]$} & - & - \\
\hline
\end{tabular}

* Extracted for absolute normalization of relative cross sections using SAID [22] partial-wave analysis parametrizations; see e.g. [14, 15].

The photoproduction reaction $\gamma p \rightarrow \eta p$ is a powerful tool in the study of baryon

\author{
CP870, Intersections of Particle and Nuclear Physics: $9^{\text {th }}$ Conference, \\ edited by T. M. Liss \\ (c) 2006 American Institute of Physics 978-0-7354-0368-0/06/\$23.00
}


resonances, since the reaction provides an "isospin filter" to the nucleon response, as $\eta N$ final states can originate only from isospin $I=1 / 2$ systems. Differential cross sections have been measured at CLAS with tagged real photons in the energy range of $W=1.51$ to $2.13 \mathrm{GeV}$ [14]. The data provide the first extensive angular distribution measurements for the process above $W=1.75 \mathrm{GeV}$. Comparison with preliminary results from a constituent quark model [23] support the suggestion that a third $S_{11}$ resonance with mass $\sim 1.8 \mathrm{GeV}$ couples to the $\eta N$ channel. Also the $\gamma p \rightarrow \eta^{\prime} p$ reaction has been studied [15]. The results, for energies from $W=1.94$ to $2.25 \mathrm{GeV}$, possess much greater accuracy than previous measurements. Analyses of these data suggest for the first time the coupling of the $\eta^{\prime} N$ channel to both the $S_{11}(1535)$ and $P_{11}(1710)$ resonances, known to couple strongly to the $\eta N$ channel in photoproduction on the proton, and the importance of $J=3 / 2$ resonances in the process.

High-statistics cross sections for the reactions $\gamma p \rightarrow K^{+} \Lambda$ and $\gamma p \rightarrow K^{+} \Sigma^{0}$ have been measured using CLAS for center-of-mass energies $W$ between 1.6 and $2.53 \mathrm{GeV}$, and for $-0.85<\cos \theta_{K^{+}}^{\text {c.m. }}<+0.95$ [17]. In the $K^{+}$channel the data confirm a resonance-like structure near $W=1.9 \mathrm{GeV}$ at backward kaon angles. The position and width of this structure change with angle, indicating that more than one resonance is likely playing a role. The $K^{+} \Lambda$ channel at forward angles and all energies is well described by a $t$ channel scaling characteristic of Regge exchange, whereas the same scaling applied to the $K^{+} \Sigma^{0}$ channel is less successful. Several existing theoretical models are compared to the data, but none provide a good representation of the results. The analysis of these data and data from the SAPHIR and LEPS Collaborations combined with data from $\pi$ and $\eta$ photoproduction reveals evidence for new baryon resonances in this energy region [24]. A new $P_{11}$ state with mass $1840 \mathrm{MeV}$ and width $140 \mathrm{MeV}$ was observed contributing to most of the fitted reactions. The data demand the presence of two $D_{13}$ states at $1875 \mathrm{MeV}$ and, optimistically, at $2170 \mathrm{MeV}$. This analysis does not find any need for introducing additional (third or forth) $S_{11}$ states - opposed to the above mentioned study of the $\eta N$ channel.

A complete set of certain polarization observables is necessary to unambiguously determine the amplitudes of the reaction. However, polarization observables are already crucial to constrain otherwise largely unconstrained models and especially partial-wave analyses, even if such a complete set has not (yet) been obtained.

The $\Lambda$ recoil polarization in the $\gamma p \rightarrow K^{+} \Lambda$ was determined through the weak decay $\Lambda \rightarrow p \pi^{-}$[18]. It is found that the large positive $\Lambda$ polarization at backward angles, which is also energy dependent, is consistent with sizable $s$ - or $u$-channel contributions. Presently available model calculations cannot explain these aspects of the data. The CLAS collaboration has also completed first measurements of the double polarization observables $C_{x}$ and $C_{z}$ for the reactions $\gamma p \rightarrow K^{+} \Lambda$ and $\gamma p \rightarrow K^{+} \Sigma^{0}$ [19]. $C_{x}$ and $C_{z}$ are the beam-recoil polarization asymmetries measuring the polarization transfer from incoming circularly polarized photons to outgoing hyperons along two directions in the reaction's production plane. The $\Lambda$ is found to be nearly maximally polarized along the direction of incident photon's polarization for forward-going kaons. Polarization transfer to the $\Sigma^{0}$ is different from the $\Lambda$ case.

Beam-helicity asymmetries for the two-pion-photoproduction reaction $\vec{\gamma} p \rightarrow p \pi^{+} \pi^{-}$ have been studied for the first time between $W=1.35$ and $2.30 \mathrm{GeV}$ [20]. The experiment was performed with circularly polarized tagged photons incident on an unpolarized 
hydrogen target. The large values for this single polarization observable $I^{\odot}$ [25] exhibit strong sensitivity to the kinematics and dynamics of the reaction. The data, compared with the results of various phenomenological model calculations, show that these models currently do not provide an adequate description for the behavior of this new observable.

The high-quality data presented here challenge models and raise presently more questions than they can answer. All of them point to the need of a comprehensive data set of polarization observables and the need to take all the coupled reaction channels into account. The CLAS frozen-spin target program combines a series of meson photoproduction experiments $[26,27,28,29,30]$ to measure single- and double-polarization observables with polarized photons on a longitudinally or transversely polarized proton (butanol) target. The data will greatly constrain partial-wave analyses and reduce modeldependent uncertainties in the extraction of nucleon resonance properties, providing a new benchmark for comparisons with QCD-inspired models.

The analyses of these data are expected to be carried out by the established partialwave analysis groups (e.g. [22]) or the newly established Excited Baryon Analysis Center (EBAC) at Jefferson Lab.

\section{ACKNOWLEDGMENTS}

Authored by The Southeastern Universities Research Association, Inc. under U.S. DOE Contract No. DE-AC05-84150. The U.S. Government retains a non-exclusive, paidup, irrevocable, world-wide license to publish or reproduce this manuscript for U.S. Government purposes.

\section{REFERENCES}

1. B.A. Mecking et al., Nucl. Instrum. Methods A503, 513 (2003).

2. M. Battaglieri et al., Phys. Rev. Lett. 87, 172002 (2001).

3. M. Battaglieri et al., Phys. Rev. Lett. 90, 022002 (2003).

4. E. Anciant et al., Phys. Rev. Lett. 85, 4682 (2000).

5. C. Djalali, these Proceedings.

6. M. Ungaro, these Proceedings.

7. H. Egiyan, these Proceedings.

8. S. Stepanyan et al., Phys. Rev. Lett. 91, 252001 (2003).

9. V. Kubarovsky et al., Phys. Rev. Lett. 92, 032001 (2004).

10. M. Battaglieri et al., Phys. Rev. Lett. 96, 042001 (2006).

11. B. McKinnon et al., Phys. Rev. Lett. 96, 212001 (2006).

12. S. Niccolai et al., Phys. Rev. Lett. 97, 032001 (2006).

13. R. DeVita et al., Phys. Rev. D 74, 032001 (2006).

14. M. Dugger et al., Phys. Rev.Lett. 89, 222002 (2002).

15. M. Dugger et al., Phys. Rev. Lett. 96, 062001 (2006).

16. F.J. Klein et al., Nucl. Phys. A 754, 321c (2005).

17. R. Bradford et al., Phys. Rev. C 73, 035202 (2006).

18. J.W.C. McNabb et al., Phys. Rev. C 69, 042201(R) (2004).

19. R. Bradford and R.A. Schumacher, Proc. of NSTAR 2005 Workshop; nucl-ex/0602004.

20. S. Strauch et al., Phys. Rev. Lett. 95, 162003 (2005).

21. J.W. Price et al., Phys. Rev. C 71, 058201 (2005).

22. R.A. Arndt, W.J. Briscoe, I.I. Strakovsky, R.L. Workman, and M.M. Pavan, Phys. Rev. C 69, 035213 (2004). 
23. B. Saghai, Proc. NSTAR 2004 (World Scientific, 2004); nucl-th/0408054.

24. A.V. Sarantsev et al., Eur. Phys. J. A 25, 441 (2005).

25. W. Roberts and T. Oed, Phys. Rev. C 71, 055201 (2005).

26. Jefferson Lab experiment E02-112, "Search for Missing Nucleon Resonances in Hyperon Photoproduction", P. Eugenio, F. Klein, and L. Todor, spokespeople.

27. Jefferson Lab experiment E03-105, "Pion Photoproduction from a Polarized Target", N. Benmouna, W. Briscoe, G. O'Rielly, I. Strakovski, and S. Strauch, spokespeople.

28. Jefferson Lab experiment E04-102, "Helicity Structure of Pion Photoproduction", D. Crabb, M. Khandaker, and D. Sober, spokespeople.

29. Jefferson Lab experiment E05-012, "Measurement of polarization observables in $\eta$-photoproduction with CLAS”, M. Dugger and E. Pasyuk, spokespeople.

30. Jefferson Lab experiment E06-013, "Measurement of $\pi^{+} \pi^{-}$Photoproduction in Double-Polarization Experiments using CLAS”, M. Bellis, V. Credé and S. Strauch, spokespeople. 